\title{
Development of a Wavelet-ANFIS based fault location system for underground power cables
}

\author{
H. S. Zawaira ${ }^{1}$, G. N. Nyakoe $^{2}$, C. M. Muriithi ${ }^{3}$ \\ ${ }^{I}$ Pan African University, Institute for Basic Sciences, Technology and Innovation, P. O. Box 62000-00200, \\ Nairobi, Kenya, \\ ${ }^{2}$ Jomo Kenyatta University of Agriculture and Technology P. O. Box 62000-00200, Nairobi, Kenya, \\ ${ }^{3}$ Jomo Kenyatta University of Agriculture and Technology P. O. Box 62000-00200, Nairobi, Kenya,
}

\begin{abstract}
In the past decade, electricity demand has increased rapidly in metropolitan areas. All over the world, large scale underground power cable installations networks are replacing overhead transmission lines due to environmental concerns in densely populated areas. Underground cable systems are manufactured to have long life with reliability. However, the useful life span of these cables is not infinite. The increase in failure rates and system breakdowns on older underground power cables are now adversely impacting system reliability and many losses involved; therefore it is readily apparent that necessary action has to be taken to manage the consequences of this trend. In this paper, a method that combines wavelets and Neuro-fuzzy technique for fault location and identification is proposed. A $10 \mathrm{~km}, 34.5 \mathrm{KV}, 50 \mathrm{~Hz}$ power transmission line model was developed and different faults and locations simulated, and then certain selected features of the wavelet transformed signals were extracted to develop an ANFIS for fault location. Comparison of the ANFIS output values and the actual values show that the percentage error was established to be less than $1 \%$. Thus, it can be concluded that the wavelet-ANFIS technique is accurate enough to be used in identifying and locating underground power line faults.
\end{abstract}

Keywords: ANFIS, Fault location, Fault types, Underground cables and Wavelet transform.

\section{Introduction}

In electrical power systems, transmission and distribution lines are pivotal links that accomplish the continuity of service from the generating plants to the end users. For the past 5 decades, electric power systems have rapidly grown [1]. Transmission lines are improving in length and capacity in terms; voltage levels and power being transmitted. These lines however are susceptible to faults which emanate from factors including insulation breakdown, short circuits and defects within the insulation of the cables. In most cases, electrical faults manifest themselves as mechanical damage which must be repaired urgently before returning the line to service [2]. The longer the power outages, the greater the damage is caused. Therefore, quick identification of fault location and restoration of power is essential for all utilities.

The speedy and precise fault location plays a pivotal role in accelerating system restoration, reducing outage time and improving system reliability [1]. Accurate location of transmission line faults has been a subject of interest to electric engineers and utilities. The major reason for this interest is mainly because accurate location of fault can reduce time required for bringing back service to customers [3]. In the past, utilities had to rely on sending staff to inspect the transmission line, which required several hours to find the actual location. A very high degree of accuracy is thus required which of cause is difficult to achieve using conventional techniques. In order to fix the fault quickly, finding the accurate location of fault point is the key [4]. In transmission and distribution systems, underground cables have mostly been used. This is because they are more secure than overhead lines and cannot be damaged by lightning or storms. These underground cable systems are manufactured to have a long life with reliability.

However, as the cables age, they start to develop internal faults, which in the long run will result into a full blown fault [5]. Internal failures in underground cables result from gradual weakening or deterioration of the insulation materials between core and sheath. Voids and impurities in the insulation material or between boundaries of different material, can initiate a process called treeing which leads to insulation breakdown [6]. When the insulation breaks, an electric arc forms a low impedance path between the cable's core and sheath allowing a large fault current to start flowing resulting in protection system disconnection. When the protection system disconnects, the fault either develops into a series fault or remain as a shunt fault [7].

To improve the reliability of a distribution system, accurate identification of a faulted segment is required in order to reduce the interruption time during fault. Therefore, a rapid and accurate fault detection method is required to accelerate system restoration, reduce outage time, minimize financial losses and significantly improve the system reliability and ensure customer power quality. Conventionally, methods that have been used for identifying and locating the cable defects, were time consuming and inefficient. This led to 
the introduction of better techniques of fault identification such as Time Domain Reflectometer (TDR), Discrete Wavelet Transformation (DWT) and artificial intelligence based methods. However, in order to further improve the power quality and reduction in power outages, more research needs to be done to accurately detect the fault location. The quick restoration of power is necessary for reliable operation of power system equipment and customer satisfaction. This study aimed at developing an underground cable fault identification and location system by use of artificial intelligence and wavelet analysis. This paper builds upon previously presented methods to design a fast and accurate method that can identify and locate faults in an underground power cable. The proposed method uses wavelet-ANFIS combined approach. In the next section, a development of transmission line simulation model is presented. In the subsequent sections, fault identification approach, design of the wavelet-ANFIS and, simulation results and their discussion are presented.

\section{Transmission Line Simulation Model}

The basic structure of the transmission line consists of the generator, the transmission system and a constant load. Figure 1 shows the basic structure of the system considered in developing the model of the transmission line used for the simulations. From the model, actual system parameters were used to come up with a MATLAB model that was simulated to generate fault signals. The development of the transmission line was done under MATLAB/SIMULINK environment. The line considered is a $10 \mathrm{~km}, 34.5 \mathrm{KV}, 50 \mathrm{~Hz}$ underground power cable. The system analysed post fault conditions and all the simulations done were to generate fault signals which were used to identify and locate the faults and generate a database. The fault was created after every $0.1 \mathrm{~km}$, with a simulation time of $0.0001 \mathrm{~s}$, sample time $=0$, resistance per unit length $=0.022 \Omega$, inductance per unit length $=0.079 \mathrm{mH}$ and capacitance per unit length $=0.038 \mu \mathrm{F}$. A $0.1 \mathrm{~km}$ distance was chosen so as to create a bigger database that was used in the development and designing of an ANFIS which is reliable and accurate in distance estimation. The system was considering a constant load.

\section{Fault Identification Approach}

Fault identification was done in post fault conditions. For the system to identify, the fault was assumed to have occurred already when the simulation was initiated. The nature of the fault and the specification of the fault were changed in the three phase fault tool box. This enables the system to have different types of fault and at different locations to cover wide scenarios which might affect the transmission system. Because transmission lines can experience single line or double phase or three phase faults, the faults were also introduced at these different locations with different behaviours. After the transmission line had been designed, the system was simulated to identify what type of fault has affected the system and on which phase. In achieving that, the initial stage was to analyse the signals of the transmission line so as to identify which phase(s) have been affected. Simulated cable data from the phase which has a fault was transferred to Wavelet Analysis in MATLAB/SIMULINK environment for data processing and analysis.

The transmission line model system was simulated to identify the type of fault on the affected phase. The system was designed with a means of observing all the phases on the transmission line on one platform. To distinguish the faults, different wave forms were observed. For a single line fault, only one phase had a lower magnitude of voltage as compared to the other two. In a double phase fault, two of the signals with faults indicated some deviations from the expected wave form. When the type of the fault had been established, fault data, which can be referred to as signal $\mathbf{s}$ from the line was analyzed under wavelet transform to extract useful information pertaining to the fault.

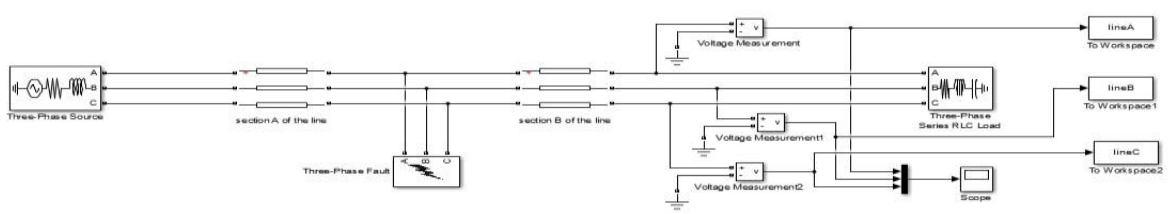

Figure 1 Line model

\section{Fault Location Using Discrete Wavelet Analysis}

To enable fault location along the transmission line, the initial stage is to analyze the fault signals so as to identify the affected phase(s). Simulated line data from the affected phase is transferred to Discrete Wavelet Transformation environment for data processing and analysis. To accomplish the task, features of the line voltage signals are extracted based on discrete wavelet transform with daubechies4 (db4) as the mother wavelet. Daubechies wavelets, are a family of orthogonal wavelets defining a discrete wavelet transform and 
characterized by a maximal number of vanishing moments for some given support. With each wavelet type of this class, there is a scaling function which generates an orthogonal multi-resolution analysis. Db4 was chosen because it works best for fast transients. The feature extracted from the line is an index representing the location of the fault, represented by spikes on the DWT. Analysing signal $\mathbf{s}$ under discrete wavelet analysis, involves passing the signal through a number of filters. Firstly the sample signal $\mathbf{s}$ is passed through a low pass filter which has an impulse response $\mathbf{g}$ resulting in a convolution of the two. Passing the signal under low pass filter enables the extraction of approximation coefficients of the signal to identify changes in signal properties. After the first decomposition, the signal had halved the time resolution since only half of each filter output characterises the signal. The two outputs each have half the frequency band of the input so the frequency resolution has been doubled. Depending with the level to which the signal has to be analysed, the process of passing the new signal over the low pass and high pass filter will continue until the level of analysis has been achieved. The levels depends with the user. At every level, the signal will be passed through high and low pass filter and the signal resolution will at each stage also double from the previous resolution. This will help the signal to be clearly viewed and to remove any noise which might be in the signal or any disturbance which might occur. At every level, a certain amount of noise and small disturbances will be eliminated making the signal clearer to analyse and to expose the changes in the signal parameters.

\section{ANFIS Model For Fault Location}

The database created after wavelet analysis for different faults and locations was used as the input in the development of an ANFIS. Depending on the knowledge of the system, there are basically three types of estimation models that can be used to solve such a problem. Black box being one of them is very useful when the primary concentration is in fitting the data irrespective of the particular mathematical structure of the model. Since black-box modelling is usually a trial-and-error process, the database which was created in wavelet analysis, was used to train, design and test the ANFIS. The end result was to develop a model which can accurately estimate the fault location given the fault signals from the transmission line. Figure 2 represents the overall structure of the ANFIS developed.

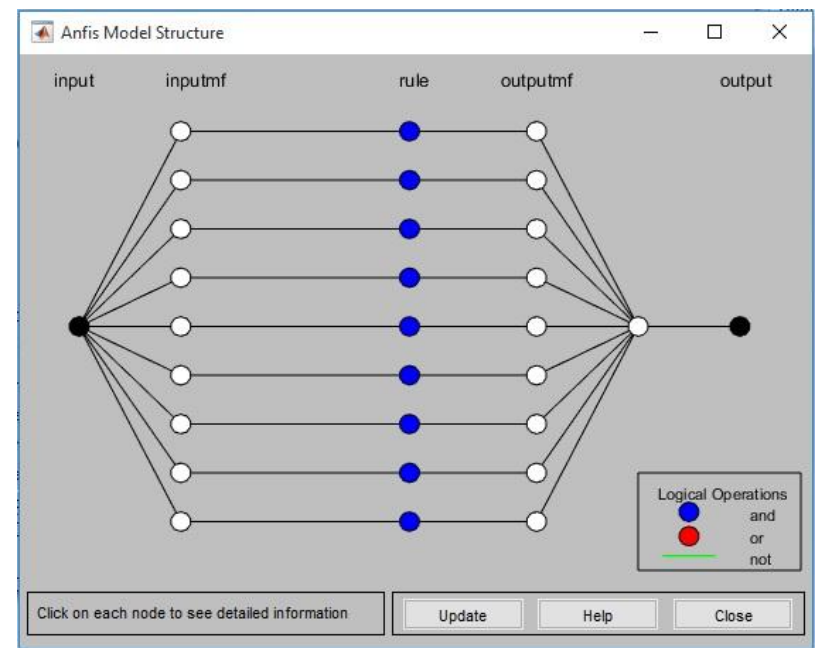

Figure 2 ANFIS model structure

\section{Results And Discussion}

To identify the phase which has been affected, the system was designed to print out all the phases and their behaviour.

\subsection{Single phase fault}

Figure 3 represents three-phase voltage signals for Line A, Line B and Line C and their detail coefficients at single phase fault condition. From Figure 3, it can be observed that Line A has been affected because of the change in the signal. From the observation, it can be identified that the system has suffered a single phase fault because of the one line which has shown a deviation from the expected. The fault in line A affects the behaviour of other lines. This can be observed by the small deviations observed on line B and Line $\mathrm{C}$. Because the system initially started as a balanced three phase system, when one of the lines has been affected, it has interference with the other lines. The straight line displayed by all signals represents the initiation time for the simulation. The spike appearing at the beginning of the signal represents the current initiation stage. The 
second spike indicates the fault location. The fault appears on the system at a specific time which after wavelet analysis shows the location of the fault.

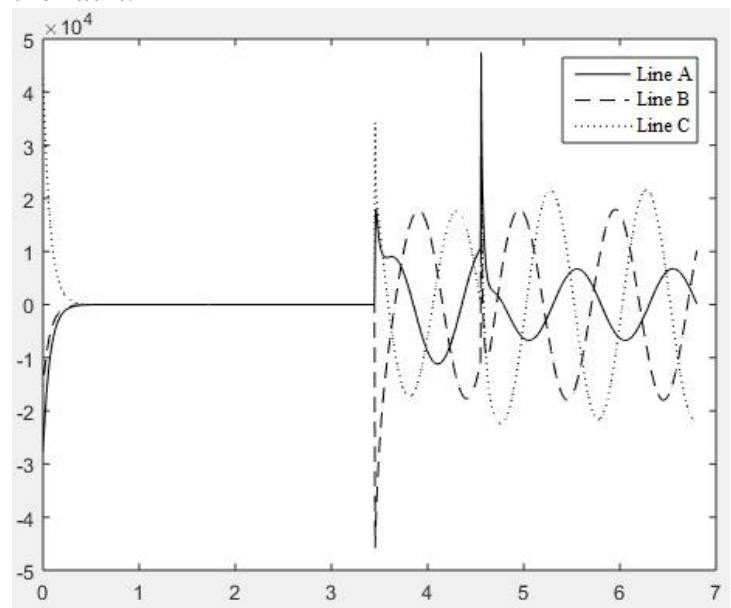

Figure 3 Fault identification for single phase fault

\subsection{Double line fault}

Figure 4 represents three-phase voltage signals for Line A, Line B and Line C and their detail coefficients at double phase fault condition. It can be observed from Figure 4 that Line A and Line B have both been affected because of the change in the amplitude of the two signals for line A and B and the introduction of the two spikes. From the figure, both the amplitudes of Line A and Line B have been affected, indicating a double phase fault and the signals have been merged into one. This indicates that the system has lost its symmetry and the two affected lines are starting to behave like the same line, because of the faults in them. Magnitude of signal from line $\mathrm{C}$ has been affected because of the faults in the other two lines. This can be observed by small changes observed on line $\mathrm{C}$. The straight line displayed by all signals represents the initiation time for the simulation. The spike appearing at the beginning of the signal represents the current initiation stage in the transmission line. The second spike indicates the fault initiation stage. The fault appears on the system at a specific time which after wavelet analysis shows the location of the fault.

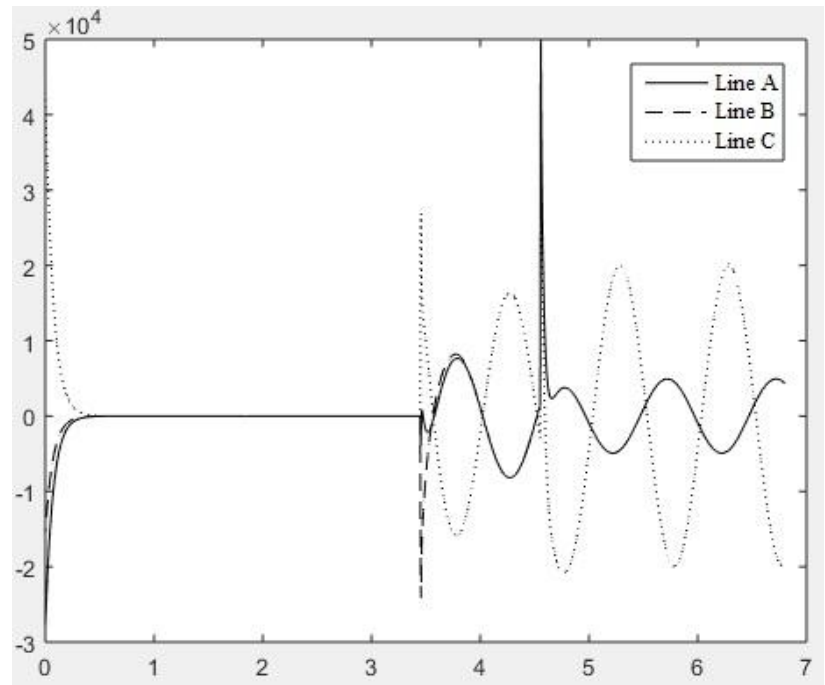

Figure 4 Fault identification for double phase fault

\subsection{Discrete wavelet transformation}

On Figure 5, the signal has been analyzed up to level 5 of analysis, indicated by $\mathrm{d} 1-\mathrm{d} 5$ on the vertical axis of Figure 5.The levels indicated by d1 - d5 represents the decomposition levels. On the first decomposition d1, the signal had halved the time resolution since only half of each filter output characterises the signal. At every level, the resolution is twice that of the previous scale. The $s$ in the figure represents the original signal obtained from the underground cable data. The a5 represents the approximate details of the signal at level 5 . The nature of the plot of detailed coefficients at level 1 reveals a sharp spike which corresponds to the fault initiation process. According to DWT theory, the spikes represent the highest frequency within the fault signal, but it is 
also however, not practical to identify a fault based on these spike only since such spikes will occur every time there is a sudden change in the cable current signal. So for precise and accurate fault location, all the levels were observed to identify consistence of spike location on all the levels. Under wavelet analysis, the location appeared as an index or number as a result of the partitions within wavelet, instead of the time variable. The index was used in ANFIS to identify the location of the fault. The ANFIS was developed in a way that it makes use of the wavelet index to locate the fault in terms of distance.

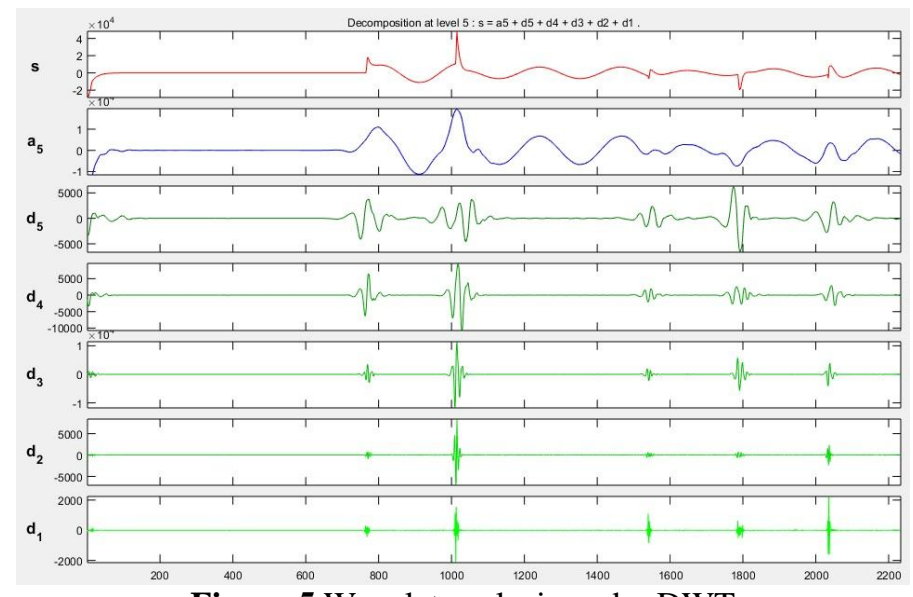

Figure 5 Wavelet analysis under DWT

\subsection{ANFIS for fault location}

The ANFIS graphic user interface was obtained for fault location. On the system, the data obtained from wavelet transform was used to identify the location of the fault. To test the efficiency and establish the accuracy of the system, a number of simulations had to be done and identify how the system will perform. Using equation 1 to identify the accuracy of the system, Table 1 is shows the results of the simulations done at random fault locations selected on the line to establish the accuracy of the ANFIS.

$\%$ Error $=\frac{\text { Actual distance }- \text { Calculated distance }}{\text { total faulty section length }} * 100$

From Table 1, it can be observed that the general difference between the simulated values and the actual fault locations are around 0.1 or -0.08 . If expressed as a percentage error from a specific line segment as shown in Figure 6, in other sections, the percentage error is big.

Table 1 Test values and error estimation

\begin{tabular}{|l|l|l|l|}
\hline & & & \\
\hline $\mathbf{1 . 4 5}$ & 1.53 & -0.08 & -5.52 \\
\hline $\mathbf{1 . 9 6}$ & 1.99 & -0.03 & -1.53 \\
\hline $\mathbf{2 . 0 3}$ & 2.09 & -0.06 & -2.96 \\
\hline $\mathbf{2 . 6 5}$ & 2.55 & 0.10 & 3.77 \\
\hline $\mathbf{3 . 1 4}$ & 3.14 & 0.00 & 0.00 \\
\hline $\mathbf{3 . 9 0}$ & 3.90 & 0.00 & 0.00 \\
\hline $\mathbf{4 . 2 1}$ & 4.26 & -0.05 & -1.19 \\
\hline $\mathbf{4 . 7 3}$ & 4.73 & 0.00 & 0.00 \\
\hline $\mathbf{5 . 3 0}$ & 5.29 & 0.01 & 0.19 \\
\hline $\mathbf{5 . 9 1}$ & 5.91 & 0.00 & 0.00 \\
\hline $\mathbf{6 . 4 3}$ & 6.44 & -0.01 & -0.16 \\
\hline $\mathbf{6 . 6 8}$ & 6.69 & -0.01 & -0.15 \\
\hline $\mathbf{7 . 3 2}$ & 7.33 & -0.01 & -0.14 \\
\hline $\mathbf{7 . 5 6}$ & 7.56 & 0.00 & 0.00 \\
\hline $\mathbf{8 . 4 5}$ & 8.43 & 0.02 & 0.24 \\
\hline $\mathbf{8 . 8 4}$ & 8.83 & 0.01 & 0.11 \\
\hline $\mathbf{9 . 3 0}$ & 9.29 & 0.01 & 0.11 \\
\hline $\mathbf{9 . 6 8}$ & 9.68 & 0.00 & 0.00 \\
\hline Average & & & $\mathbf{- 0 . 4 0}$ \\
\hline
\end{tabular}

The system was generally accurate across different segments of the line as shown in Figure 6 with the bottom $3 \mathrm{~km}$ from the sending end constituting most of the greatest errors as compared to the upper region. On distances less than $3 \mathrm{~km}$, the \% error is great because the overall distance being considered is small. Regardless 
of the actual error being small, it affects the overall \% error of the system. Generally the system was showing errors along the whole section of the cable. This is mainly cause by the training data used. Basing on the test values, the system is accurate enough with an accuracy of $99.6 \%$, to be used for fault location and identification.

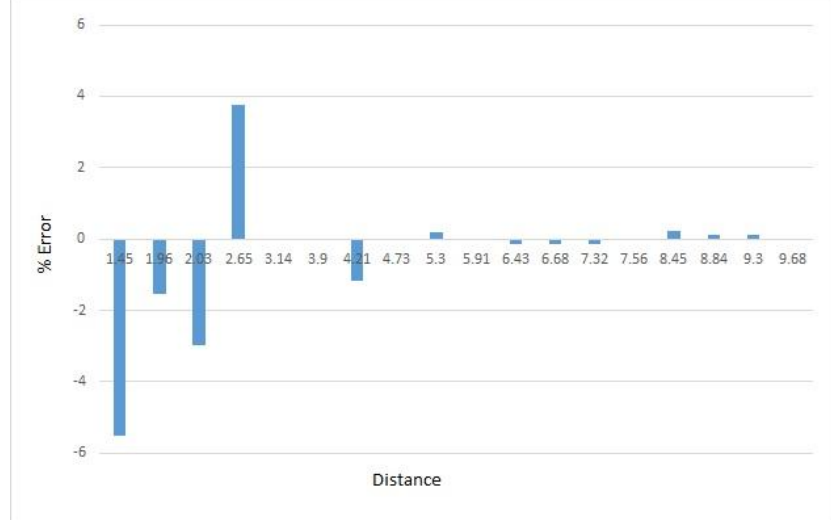

Figure 6 Distance and \% error graph

\section{Conclusion}

Simulations for the transmission line for $34.5 \mathrm{kV}, 10 \mathrm{~km}$ transmission line were performed using MATLAB/SIMULINK. In this work, the proposed method used wavelet decomposition which provided more features about the signal. The extracted information about the signal was used to develop an ANFIS which was later used to identify the fault location. The ANFIS managed to locate the fault with an accuracy of 99.6\%. The main aim of this study was to develop a method for fault location for underground power cables based on wavelet-ANFIS technique. Performance of Adaptive-Neuro Fuzzy Inference System for fault location was assessed and results were obtained. Finally it was shown that the proposed method for underground power cable fault location based on wavelet-ANFIS technique is accurate enough to be used in detection of transmission line fault.

\section{References}

[1] M. Mirzaei, M. A. A. Kadir, E. Moazami, and H. Hizam, "Review of Fault Location Methods for Distribution Power System," Australian Journal of Basic and Applied Sciences, vol. 3, no. 3, 2009.

[2] M. Mashikian and A. Szatkowski, "Medium Voltage Cable Defects Revealed by Off-LinePartial Discharge Testing at Power Frequency," IEEE Electrical Insulation Magazine, vol. 22, no. 4, pp. 24-32, July/August 2006

[3] G. Banu1 and S. Suja2, "Fault location technique using GA-ANFIS for UHV line," Archives of Electrical Engineering, vol. 63, no. 2, pp. 247-262, 2014.

[4] Chang and Jin, "Single ended travelling wave based fault location using Discrete Wavelet Transform, ” uknowledge.uky.edu, no. 58, 2014.

[5] M. B. Eteiba, W. I. Wahba, and S. Barakat, "ANFIS Approach for Locating Faults in Underground Cables," International Journal of Electrical, Computer, Energetic, Electronic and Communication Engineering, vol. 8, no. 6, 2014

[6] J. Densley, "Ageing mechanisms and diagnostics for power cables, an overview," IEEE Electrical. Insulation. Mag, vol. 17, no. 1, pp. 14-22, 2001.

[7] J. Altamirano, T. Andrews, M. Begovic, Y. del Valle, R. Harley, J. C. H. Mejiaand, and T. J. Parker, "Diagnostic testing of underground cable systems (cable diagnostic focused initiative)," NEETRAC project numbers: 04-211/04-212/09-166, December 2010. 